\title{
Psychological approaches may improve oral hygiene behaviour
}

\section{What interventions improve adherence to oral hygiene instruction in adults who have periodontal disease?}

\author{
Renz A, Ide M, Newton T, Robinson PG, Smith D. Psychological \\ interventions to improve adherence to oral hygiene instructions \\ in adults with periodontal diseases. Cochrane Database Syst ReV \\ 2007; issue 2
}

Data sources The Cochrane Oral Health Group's Trials Register, Cochrane Central Register of Controlled Trials, Medline, Embase, Psychinfo, Ingenta and Cumulative Index to Nursing and Allied Health Literature were searched, along with the reference lists from relevant articles. The authors of eligible trials were contacted to identify further studies and obtain additional information. Four journals were searched by hand (Journal of Health Psychology, Psychology and Health, Psychology Health and Medicine, Health Psychology - Update). No language restriction was applied.

Study selection Randomised controlled trials were selected that tested the effectiveness of interventions based on psychological models, comparing them with educational, attention or no active intervention controls, to improve adherence to oral hygiene in adults with either gingivitis or periodontitis. Only trials with at least 3 weeks' followup were included. Interventions aimed at encouraging smoking cessation were not included.

Data extraction and synthesis Titles and abstracts of studies that were potentially relevant to the review were independently screened by two review authors. Those that were clearly ineligible were rejected. For the remaining studies, the full paper was reviewed by two review authors and, where necessary, further information was sought from the author to verify eligibility. The quality of included studies was assessed using standard criteria.

Results Four studies (of 344 participants) were found in which a psychological model or theory had been explicitly used as the basis for the design of the intervention. The overall quality of trials was low. Because of the heterogeneity of studies, both in terms of outcome measures and psychological models adopted, a meta-analysis was not possible. The four studies adopted four different theoretical frameworks, although there was some overlap in that three of the studies incorporated elements of Operant and Classical Conditioning.
Address for correspondence: Luisa Fernandez Mauleffinch, Cochrane Oral Health Group, Manchester Dental Education Centre, School of Dentistry, University of Manchester, Higher Cambridge Street, Manchester, M15 6FH, UK.
Psychological interventions resulted in improved plaque scores compared with no-intervention groups, and in one study compared with an attention control group. One study found decreased gingival bleeding in the active intervention group but no change in pocket depth or attachment loss after 4 months. Psychological interventions were associated with improved self-reported brushing and flossing in both the studies that assessed these behaviours. Only one study explored the impact of psychological interventions on beliefs and attitudes: the psychological intervention, when compared with educational and nointervention controls, showed improved self-efficacy beliefs in relation to flossing, but no effect on dental knowledge or self-efficacy beliefs in relation to tooth brushing.

Conclusions There is some evidence from low-quality studies that psychological approaches to behaviour management, such as the use of reinforcement, goal setting and the provision of feedback, can improve oral hygiene and oral hygiene-related behaviours. The design of the interventions was weak and limited, however, and ignored key aspects of the theories. All the studies reported here also relied upon the provision of guidance by personnel other than the practitioner. There is no evidence that the use of such an approach by the practitioner with his or her patients would be beneficial. Thus, there is a need for greater methodological rigour in the design of trials in this area.

\section{Commentary}

This Cochrane Review, as with most of the others, is an outstanding systematic review with a forgettable clinical bottom line. The Plain Language Summary finishes with: "...there is a need for greater methodological rigour in the design of trials in this area." Were this heaven, trials would be perfect, but we live in an imperfect world.

With the tremendous amount of time and resources required to initiate, manage, complete, review and edit a Cochrane Review, and the commitment of authors to update reviews, it might be hoped that authors and editors would consider more selective identification of topics of critical clinical importance, and base this selection on substantiated evidence. The problem here is clear in the Background section, where the second and third paragraphs begin with the following unsubstantiated assertions, "It is widely accepted that periodontal disease is caused by inflammatory responses in gum tissue following the accumulation of dental bacterial plaque" and, "Treatment of periodontal disease is mainly based around effective self care, largely in the form of oral hygiene."

The review then goes on to examine the effect of psychological models of oral hygiene behaviour-change in periodontal patients. There are two key elements to consider (and a host of intervening elements). From a causative perspective, periodontal disease is a bacterial infection that can be effectively treated with metronidazole plus amoxicillin, independently of any professional care or oral hygiene instruction. ${ }^{1}$ Second, from a preventive perspective, brushing and flossing are difficult skills to teach, carry out and quantify, 
even in compliant individuals. ${ }^{2,3}$ Given these facts, and the recent trials indicating that a mouthwash can be as, or more effective, than brushing, flossing, or brushing and flossing, ${ }^{4-6}$ one might consider investing time and resources on topics of greater importance (eg, clinician behaviour).

\section{Richard Niederman}

Forsyth Institute and Boston University Goldman School of Dental Medicine, Boston, Massachusetts, USA

1. Lopez NJ, Socransky SS, Da Silva I, Japlit MR, Haffajee AD. Effects of metronidazole plus amoxicillin as the only therapy on the microbiological and clinical parameters of untreated chronic periodontitis. J Clin Periodontol 2006; 33:648-660.
2. Niederman R, Sullivan TM. Oral Hygiene Skill Achievement Index I. J Periodontol $1981 ; 52: 143-149$.

3. Niederman R, Sullivan TM, Weiss D, Morhart R, Robbins W, Maier D. Oral Hygiene Skill Achievement Index II. J Periodontol 1981; 52:150-154.

4. Charles CH, Sharma NC, Galustians HJ, Qaqish J, McGuire JA, Vincent JW. Comparative efficacy of an antiseptic mouthrinse and an antiplaque/ antigingivitis dentifrice. A six-month clinical trial. J Am Dent Assoc 2001; 132:670-675.

5. Sharma NC, Charles CH, Qaqish JG, Galustians HJ, Zhao Q, Kumar LD. Comparative effectiveness of an essential oil mouthrinse and dental floss in controlling interproximal gingivitis and plaque. Am J Dent 2002; 15:351-355.

6. Bauroth K, Charles CH, Mankodi SM, Simmons K, Zhao Q, Kumar LD. The efficacy of an essential oil antiseptic mouthrinse vs. dental floss in controlling interproximal gingivitis: a comparative study. J Am Dent Assoc 2003; 134:359-365.

Evidence-Based Dentistry (2007) 8, 5-6. doi:10.1038/sj.ebd.6400487 